This item was submitted to Loughborough's Research Repository by the author.

Items in Figshare are protected by copyright, with all rights reserved, unless otherwise indicated.

Wave interaction with two-dimensional bodies floating in a two-layer fluid: uniqueness and trapped modes

PLEASE CITE THE PUBLISHED VERSION

LICENCE

CC BY-NC-ND 4.0

REPOSITORY RECORD

Kuznetsov, N., M. Mclver, and P. Mclver. 2019. "Wave Interaction with Two-dimensional Bodies Floating in a Two-layer Fluid: Uniqueness and Trapped Modes”. figshare. https://hdl.handle.net/2134/454. 


\title{
Wave interaction with two-dimensional bodies floating in a two-layer fluid: uniqueness and trapped modes
}

\author{
By N. KUZNETSOV${ }^{1}$, M. McIVER \\ AND P. McIVER $\mathbf{R}^{2}$ \\ ${ }^{1}$ Institute of Problems in Mechanical Engineering, Russian Academy of Sciences, \\ V.O., Bol'shoy pr. 61, St Petersburg 199178, RF \\ ${ }^{2}$ Department of Mathematical Sciences, Loughborough University, \\ Loughborough, Leics, LE11 3TU, UK \\ (Received ?? and in revised form ??)
}

Existing results on the linearised water-wave problem for a homogeneous fluid are extended to the case of a two-layer fluid. In particular, the appropriate form of Maz'ya's identity is presented and used to obtain uniqueness results, and examples of geometries are constructed for which trapped modes occur.

\section{Introduction}

This paper is concerned with the linearised problems of radiation and scattering of waves by bodies floating in and/or beneath the free surface of a fluid. For a homogeneous fluid, the questions of uniqueness and the existence of trapped modes have received much attention over several decades; see chapters 1-5 of the monograph by Kuznetsov, Maz'ya \& Vainberg (2002). (Trapped modes are non-trivial solutions to the homogeneous boundary-value problem and provide examples of non-uniqueness in a nonhomogeneous problem.) However much less is known about the same questions in the case of a two-layer fluid. The aim of the present note is to demonstrate that in many respects the problem of bodies floating in a two-layer fluid is similar to that for a homogeneous fluid. For the sake of simplicity we restrict ourselves to the two-dimensional problem.

In this paper the so-called 'Maz'ya's integral identity' for a two-layer fluid is presented in $\S 3$ and used to obtain results on uniqueness in $\S 4$. Examples of structures that support trapped modes are presented in $\S 5$.

\section{Statement of the problem and the energy lemma}

A sketch of the geometry is shown in figure 1 in which $W^{(1)}$ and $W^{(2)}$ are fluid domains occupied by fluids having respectively densities $\rho_{1}$ and $\rho_{2}$, with $\rho_{2}>\rho_{1}>0$. The superscripts (1) and (2) indicate body contours confined to $L=\{-\infty<x<\infty, 0<y<d\}$ and $\mathbb{R}_{-}^{2}=\{-\infty<x<\infty, y<0\}$, respectively, and it is assumed that the lower fluid is unbounded from below. The subscript $\mathrm{i}$ indicates immersed contours within either $L$ or $\mathbb{R}_{-}^{2}$, whereas sp (ip) denotes surface-piercing (interface-piercing) contours. Smooth curves $S_{\mathrm{sp}}^{(1)}$ and $S_{\mathrm{ip}}^{(j)}(j=1,2)$ are assumed not to be tangent to $\{y=d\}$ and $\{y=0\}$ respectively; some of the curves $S_{\mathrm{sp}}^{(1)}$ may pass through the interface. The parts of the free surface of the upper fluid and the interface outside all bodies are denoted by $F$ and $I$ 


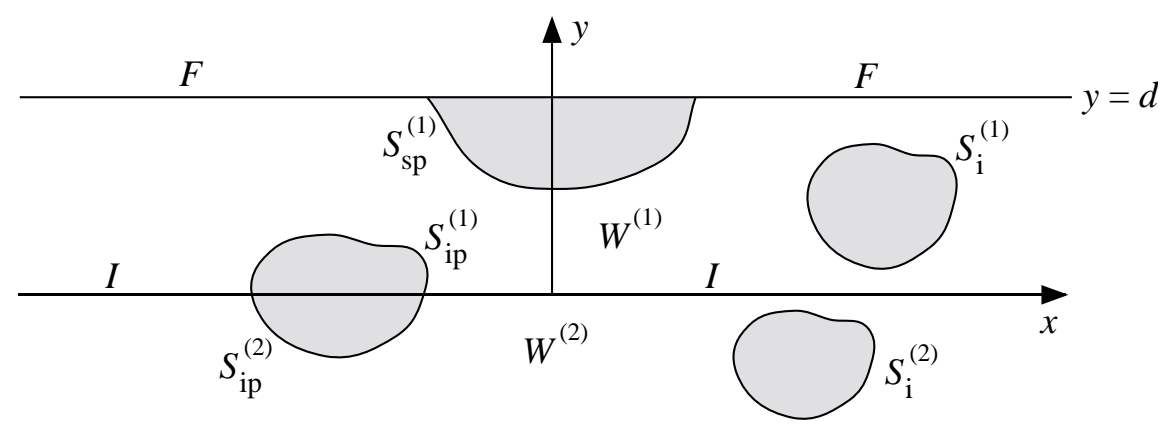

FIGURE 1. Definition sketch for bodies in a two-layer fluid.

respectively. Both fluids are assumed to be inviscid and incompressible and their motion to be irrotational so that it may be described by velocity potentials $\phi^{(1)}$ in $\overline{W^{(1)}}$ and $\phi^{(2)}$ in $\overline{W^{(2)}}$, respectively. The corresponding coupled boundary-value problem is as follows (a time dependence $\mathrm{e}^{-\mathrm{i} \omega \mathrm{t}}$ having been extracted):

$$
\begin{gathered}
\nabla^{2} \phi^{(j)}=0 \quad \text { in } \quad W^{(j)}, \quad \partial_{n} \phi^{(i)}=0 \quad \text { on } \quad S^{(j)}, \quad j=1,2 \\
\phi_{y}^{(1)}-\nu \phi^{(1)}=0 \quad \text { on } F \\
\rho\left(\phi_{y}^{(1)}-\nu \phi^{(1)}\right)=\phi_{y}^{(2)}-\nu \phi^{(2)} \text { and } \phi_{y}^{(1)}=\phi_{y}^{(2)} \text { on } I .
\end{gathered}
$$

Here $\partial_{n}$ indicates the normal derivative on $S^{(1)}=S_{\mathrm{sp}}^{(1)} \cup S_{\mathrm{i}}^{(1)} \cup S_{\mathrm{ip}}^{(1)}$ and $S^{(2)}=S_{\mathrm{ip}}^{(1)} \cup$ $S_{\mathrm{i}}^{(2)}, \nu=\omega^{2} / g>0, g$ is the acceleration due to gravity, and $\rho=\rho^{(1)} / \rho^{(2)}$ is the nondimensional measure of stratification. The homogeneous Neumann condition indicates an absence of any forcing and is used in investigations of uniqueness and trapped modes.

Usually problem (2.1)-(2.3) is supplemented by radiation conditions (formulae (2.8) and (2.9) in Linton \& McIver, 1995). However, these formulae are not given explicitly as the following assertion holds in the present case.

The energy lemma: Let $\phi^{(1)}$ and $\phi^{(2)}$ satisfy (2.1)-(2.3), radiation conditions, and the conditions that the Dirichlet integrals over certain neighbourhoods of the points of intersection of $S_{s p}^{(1)}$ and $S_{i p}^{(j)}(j=1,2)$ with respectively $F$ and I are finite. Then

$$
\sum_{j=1}^{2} \int_{W^{(j)}}\left|\nabla \phi^{(j)}\right|^{2} \mathrm{~d} x \mathrm{~d} y+\nu \int_{F}\left|\phi^{(1)}\right|^{2} \mathrm{~d} x+\nu^{-1} \int_{I}\left|\partial_{y} \phi^{(2)}\right|^{2} \mathrm{~d} x<\infty .
$$

This lemma means that trapped modes (if they exist) have both finite kinetic and finite potential energy. The proof is based on the asymptotic formulae for the Green's functions (see Linton \& McIver, 1995) and on the Green's representation formulae involving certain cut-off functions (see Kuznetsov et al., 2002, § 2.1).

We assume $\phi^{(1)}$ and $\phi^{(2)}$ to be real functions which is admissible in view of (2.4).

\section{Maz'ya's identity}

In Kuznetsov et al. (2002), chapters 2 and 3, it is demonstrated that Maz'ya's integral identity is a powerful tool for finding configurations of bodies immersed either totally or partially in a homogeneous fluid such that the uniqueness theorem is true for those geometries. In this section we generalise that identity for solutions of problem (2.1)-(2.3) 
satisfying (2.4). The starting point is Maz'ya's differential identity

$$
\begin{aligned}
2\left[(\boldsymbol{V} \cdot \boldsymbol{\nabla} u+H u) \boldsymbol{\nabla}^{2} u\right]= & 2 \boldsymbol{\nabla} \cdot[(\boldsymbol{V} \cdot \boldsymbol{\nabla} u+H u) \boldsymbol{\nabla} u] \\
& +(Q \boldsymbol{\nabla} u) \cdot \boldsymbol{\nabla} u+u^{2} \boldsymbol{\nabla}^{2} H-\boldsymbol{\nabla} \cdot\left[|\boldsymbol{\nabla} u|^{2} \boldsymbol{V}+u^{2} \boldsymbol{\nabla} H\right],
\end{aligned}
$$

which can easily be verified by direct calculation. Here $u$ is an arbitrary twice-differentiable function, $\boldsymbol{V}=\left(V_{x}, V_{y}\right)$ is a real vector field with components that are uniformly Lipschitz in a certain fluid domain, $H$ is a real function with uniformly Lipschitz first derivatives in the same domain, and

$$
Q=\left[\begin{array}{cc}
-\partial_{x} V_{x}+\partial_{y} V_{y}-2 H & -\left(\partial_{y} V_{x}+\partial_{x} V_{y}\right) \\
-\left(\partial_{y} V_{x}+\partial_{x} V_{y}\right) & \partial_{x} V_{x}-\partial_{y} V_{y}-2 H
\end{array}\right]
$$

It is easy to show that $Q$ is nonnegative definite when $H \leqslant 0$ and $\operatorname{det} Q \geqslant 0$.

Lemma (2.4) means that provided $\boldsymbol{V}^{(j)}$ and $H^{(j)}(j=1,2)$ grow sufficiently slowly as $x^{2}+y^{2} \rightarrow \infty,(3.1)$ with $u$ replaced by $\phi^{(j)}$ may be integrated over $W^{(j)}$. The resulting equality for $j=1$ is multiplied by $\rho$ and added to that for $j=2$, and the conditions

$$
V_{x}^{(1)}=V_{x}^{(2)}, \quad V_{y}^{(1)}=V_{y}^{(2)}=0, \quad H^{(1)}=H^{(2)}, \quad \partial_{y} H^{(1)}=\partial_{y} H^{(2)}=0,
$$

are imposed on $I$. After some algebra one arrives at Maz'ya's integral identity for a two-layer fluid, namely

$$
\begin{aligned}
& \rho\left\{\int_{F}\left[V_{y}^{(1)} \nu^{2}+\left(2 H^{(1)}-\partial_{x} V_{x}^{(1)}\right) \nu-\partial_{y} H^{(1)}\right]\left|\phi^{(1)}\right|^{2} \mathrm{~d} x\right. \\
& \left.-\int_{F} V_{y}^{(1)}\left|\partial_{x} \phi^{(1)}\right|^{2} \mathrm{~d} x-\nu \sum_{k=1}^{M^{(1)}}\left[\left|\phi^{(1)}(x, d)\right|^{2} V_{x}^{(1)}(x, d)\right]_{x=a_{k}^{(1)}}^{x=b_{k}^{(1)}}\right\} \\
& +\frac{1-\rho}{\nu}\left\{\int_{I}\left(2 H^{(2)}-\partial_{x} V_{x}^{(2)}\right)\left|\partial_{y} \phi^{(2)}\right|^{2} \mathrm{~d} x-\sum_{k=1}^{M^{(2)}}\left[\left|\partial_{y} \phi^{(2)}(x, 0)\right|^{2} V_{x}^{(2)}(x, 0)\right]_{x=a_{k}^{(2)}}^{x=b_{k}^{(2)}}\right\} \\
& +\sum_{j=1}^{2} \rho^{2-j}\left\{\int_{W^{(j)}}\left[\left(Q^{(j)} \nabla \phi^{(j)}\right) \cdot \nabla \phi^{(j)}+\left|\phi^{(j)}\right|^{2} \nabla^{2} H^{(j)}\right] \mathrm{d} x \mathrm{~d} y\right. \\
& \left.+\int_{S^{(j)}}\left(\left|\nabla \phi^{(j)}\right|^{2} \boldsymbol{V}^{(j)} \cdot \boldsymbol{n}+\left|\phi^{(j)}\right|^{2} \partial_{n} H^{(j)}\right) \mathrm{d} S\right\}=0 .
\end{aligned}
$$

Here $\mathbf{n}$ is the unit normal to $S^{(1)} \cup S^{(2)}$ directed into the fluid and $M^{(1)}\left(M^{(2)}\right)$ is the number of surface-piercing (interface-piercing) bodies. The left and right endpoints of the contour of the $k$ th surface-piercing body are denoted, respectively, by $\left(a_{k}^{(1)}, d\right)$ and $\left(b_{k}^{(1)}, d\right)$ and of the $k$ th interface-piercing body by $\left(a_{k}^{(2)}, 0\right)$ and $\left(b_{k}^{(2)}, 0\right)$.

Next we apply the Maz'ya integral identity and obtain several sets of conditions that guarantee that (2.1)-(2.3) have only a trivial solution.

\section{Geometries providing uniqueness}

In order to obtain geometric conditions that guarantee uniqueness, $H^{(j)}$ and $\boldsymbol{V}^{(j)}$ $(j=1,2)$ must be chosen so that all terms on the left-hand side of (3.3) are nonnegative and at least one of them is strictly positive for nontrivial $\phi^{(1)}$ and $\phi^{(2)}$; this leads to a contradiction thus proving the uniqueness theorem. Throughout this section we take $H^{(1)}=H^{(2)}=-1 / 2$. 

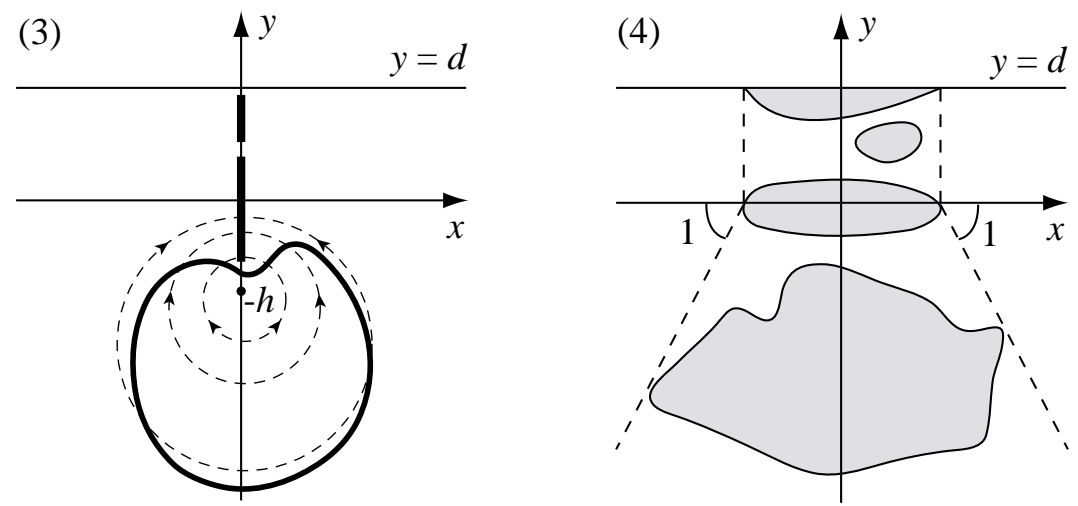

Figure 2. Bodies for which uniqueness is established in examples 3 and 4.

Example 1: Let $\boldsymbol{V}^{(1)}=\boldsymbol{V}^{(2)}=(-x, 0)$, so that conditions (3.2) hold, and let $S^{(1)} \cup$ $S^{(2)}$ be an arbitrary set of finite segments on the $y$ axis. Then all terms on the lefthand side of (3.3) vanish except for the area integrals. The latter are strictly positive for nontrivial $\phi^{(1)}$ and $\phi^{(2)}$ because

$$
Q^{(1)}=Q^{(2)}=\left[\begin{array}{ll}
2 & 0 \\
0 & 0
\end{array}\right]
$$

for our choice of $H^{(j)}$ and $\boldsymbol{V}^{(j)}(j=1,2)$. Thus the uniqueness theorem holds for a vertical barrier with gaps (intersecting or not the free surface and the interface). The same is true when, apart from finite segments, $S^{(1)} \cup S^{(2)}$ includes a semi-infinite ray extending downwards from a point $(0, c)$, where $c<d$.

Example 2: Let

$$
\boldsymbol{V}^{(1)}=\boldsymbol{V}^{(2)}= \begin{cases} \pm(b-|x|), & \pm x>b, \\ 0, & |x|<b,\end{cases}
$$

and again all of the conditions (3.2) hold (example 1 is the degenerate case $b=0$ ). Further suppose that there is both a surface-piercing and an interface piercing body whose horizontal extremes are exactly at $|x|=b$, then the uniqueness theorem holds irrespective of the presence or absence of further fully immersed bodies within $|x| \leqslant b$.

This result extends the uniqueness theorem of John (1950) for the two-dimensional water-wave problem to the case of a two-layer fluid. For the proof, considerations from example 1 must be applied when $|x|>b$. For $|x|<b$, it is sufficient to note that $Q^{(1)}$ and $Q^{(2)}$ are each the $2 \times 2$ identity matrix.

Example 3: Let $\boldsymbol{V}^{(1)}=(-x, 0)$ and $\boldsymbol{V}^{(2)}=\left(x\left(y^{2}-x^{2}-h^{2}\right) / N,-2 x^{2} y / N\right)$ where $N^{2}=\left(y^{2}-x^{2}-h^{2}\right)^{2}+4 x^{2} y^{2}$ and $h$ is a nonnegative constant. Then conditions (3.2) are satisfied and a direct but tedious calculation gives $\operatorname{det} Q=4 x^{2} h^{2} / N^{2}$ and so $Q$ is a nonnegative definite matrix as noted in $\S 3$. Let $\overline{W^{(1)}}$ either be free of bodies or contain a vertical barrier as described in example 1. Then all terms on the left-hand side of (3.3) are nonnegative except for the integral over $S^{(2)}$. The latter is also nonnegative when

$$
x\left(y^{2}-x^{2}-h^{2}\right) n_{x}-2 x^{2} y n_{y} \geqslant 0 \quad \text { on } S^{(2)},
$$

and so this inequality is a sufficient condition for uniqueness. Geometrically inequality (4.1) means that the vector field makes angles not exceeding $\pi / 2$ with the normals on $S^{(2)}$ directed into the fluid. The integral curves of the vector field are circles belonging 
to one of the coordinate lines of the bipolar system with poles at $(0, \pm h)$. A geometry satisfying (4.1) and hence guaranteeing uniqueness is shown in figure 2.

Example 4: Let $\boldsymbol{V}^{(1)}$ be the same as in example 2 and

$$
\boldsymbol{V}^{(2)}=\left\{\begin{array}{lc}
-\left[1-\pi-\theta_{-}\right](x+b, y), & -\pi \leqslant \theta_{-} \leqslant-\pi+1, \\
0, & \text { in } W_{0}, \\
-\left[1+\theta_{+}\right](x-b, y), & -\pi \leqslant \theta_{+} \leqslant 0,
\end{array}\right.
$$

where $\theta_{ \pm}=\arctan \left(y /(x \mp b)\right.$ and $W_{0}$ is the subdomain of $\mathbb{R}_{-}^{2}$ between the lines $\theta_{+}=1$ and $\theta_{-}=1-\pi$. Again all of the required properties are fulfilled and it is straightforward to show that $\operatorname{det} Q^{(2)} \geqslant 0$. Therefore this example generalises example 2 by allowing bodies fully immersed in the lower fluid to be within the region $W_{0}$ that is wider than the strip $\{-b \leqslant x \leqslant b\}$. It is also an extension of the example of Weck (1990) for a homogeneous fluid. However, unlike example 2 which is extendable to the three-dimensional case, example 4 has a straightforward generalisation only to axisymmetric geometries. Figure 2 illustrates a geometry for which uniqueness is guaranteed.

\section{Trapped modes}

In this section examples of geometries that support trapped modes are constructed by the inverse method of McIver (1996) in which non-trivial solutions to the homogeneous problem are found from singular solutions of the governing equations. Individual singular solutions radiate waves to infinity, but two solutions may be combined in such a way as to cancel these waves. The streamline pattern reveals lines which isolate the singularities and hence some streamlines may represent structures that support trapped modes.

First we summarise some of the properties of the dispersion equation for waves in twolayer fluid which is $(k-\nu)\left[\nu\left(\sigma+\mathrm{e}^{-2 k d}\right)-k\left(1-\mathrm{e}^{-2 k d}\right)\right]=0$, where $k$ is the wavenumber and $\sigma=(1+\rho) /(1-\rho)>1$; the dispersion equation has two positive roots, $k=\nu$ and $k=\nu_{0}$ (see Linton \& McIver, 1995) such that

$$
\nu \sigma<\nu_{0}<\nu(\sigma+1) /\left(1-\mathrm{e}^{-2 \sigma \nu d}\right) .
$$

\subsection{Sources}

One of the features of sources in the presence of an interface is that it is not possible to construct an isolated source for which the potential does not grow logarithmically as the distance from the source point tends to infinity (see, for example, equations (27) and (28) of Gorgui \& Kassem, 1978). Thus, to obtain from source potentials a trapped-mode potential that is bounded at infinity it is necessary to combine at least two singularities. Here attention will be restricted to the case when there are singularities on either side of the interface so that solutions singular at $(x, y)=(\xi, \pm \eta)$ are sought and the limit $\eta \rightarrow 0$ taken. The derivation follows closely that used for other singularities by Linton \& McIver (1995) and hence is omitted. The resulting singular solution is

$$
G_{0}^{(j)}(x, y ; \xi)= \begin{cases}f_{0}^{\infty}\left[A(k) \mathrm{e}^{k y}+B(k) \mathrm{e}^{-k y}\right] \cos k(x-\xi) \mathrm{d} k, & j=1, \\ f_{0}^{\infty} C(k) \mathrm{e}^{k y} \cos k(x-\xi) \mathrm{d} k & j=2,\end{cases}
$$

where $G_{0}^{(j)}$ is the potential in layer $j, f$ denotes a principal-value integral, and

$$
A(k)=-\frac{(k+\nu) \mathrm{e}^{-2 k d}}{(k-\nu) h(k)}, \quad B(k)=-\frac{1}{h(k)}, \quad C(k)=\frac{\left[k-\nu-(k+\nu) \mathrm{e}^{-2 k d}\right]}{(k-\nu) h(k)},
$$




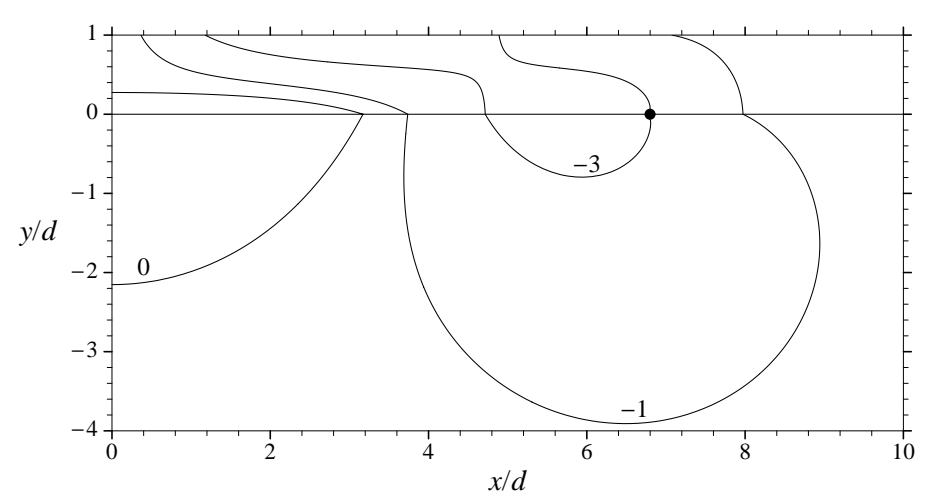

FiguRE 3. Streamline pattern for two sources on either side of interface; $m=1, n=0, \sigma=2$.

with $h(k)=(k+\nu) \mathrm{e}^{-2 k d}-k+\sigma \nu$.

It is readily shown (see Linton \& McIver, 1995) that for $j=1,2$ as $\nu|x| \rightarrow \infty$

$$
\begin{aligned}
G_{0}^{(j)}(x, y ; \xi) \sim & -\pi\left[\mathrm{R}(C: \nu) \mathrm{e}^{\nu y} \sin |\nu(x-\xi)|\right. \\
& \left.+\mathrm{R}\left(C: \nu_{0}\right) \mathrm{e}^{\nu_{0} y}\left[\frac{\nu \sigma-\nu_{0}}{\nu(\sigma-1)}+\frac{\nu-\nu_{0}}{\nu(\sigma-1)} \mathrm{e}^{-2 \nu_{0} y}\right]^{2-j} \sin \left|\nu_{0}(x-\xi)\right|\right],
\end{aligned}
$$

where $\mathrm{R}(C: \mu)$ denotes the residue of $C(k)$ at $k=\mu$. To construct trapped mode solutions the wave terms at infinity are annulled by combining two singularities as

$$
\mathcal{U}_{ \pm}^{(j)}(x, y ; \xi)=G_{0}^{(j)}(x, y ;-\xi) \pm G_{0}^{(j)}(x, y ; \xi), \quad j=1,2 .
$$

It is a simple matter to verify that the waves at infinity are annulled in $\mathcal{U}_{+}^{(j)}$ by choosing

$$
\nu_{0} \xi=(2 m+1) \pi / 2 \quad \text { and } \quad \nu \xi=(2 n+1) \pi / 2
$$

and in $\mathcal{U}_{-}^{(j)}$ by choosing

$$
\nu_{0} \xi=m \pi \quad \text { and } \quad \nu \xi=n \pi
$$

where in each case $m$ and $n$ are integers. For a given $\sigma, \nu$ and $\nu_{0}$ are chosen in the form of either (5.4) or (5.5) such that $\nu_{0} / \nu>\sigma$, see (5.1); for given $\nu_{0} / \nu, \nu_{0} d$ follows from the dispersion equation. As noted above, structures that support trapped modes are found by identifying suitable streamlines which are the level contours of the stream function $\mathcal{V}_{ \pm}^{(j)}$ corresponding to $\mathcal{U}_{ \pm}^{(j)}, j=1,2$.

A huge variety of trapping structures may be generated. One example of the streamline pattern that may be obtained by the above construction (with the sources added) is shown in Fig 3 where the position of the singularity is shown by a disc; the numbers of the streamlines denote the corresponding value of the stream function $\mathcal{V}_{+}$. The pattern is symmetric about $x=0$ so that only the pattern for $x \geqslant 0$ is shown. The streamline $\mathcal{V}_{+}=-1$ excludes the singularity from the flow field and is one example of the surface of a trapping structure. The part of this streamline in the lower fluid has a similar shape to those found in the corresponding construction for a homogeneous fluid by McIver (1996). Other closed streamlines, such as that corresponding to $\mathcal{V}_{+}=0$, may also included in an arrangement of trapping structures. On the other hand the streamline $\mathcal{V}_{+}=-3$ passes through the singularity and does not correspond to the surface of a trapping structure. 


\subsection{Dipoles}

Unlike the source potential considered above, it is possible for a dipole singularity that is bounded at infinity to exist in isolation. Dipoles have been used for constructing examples of trapped modes in a homogeneous fluid by Motygin and Kuznetsov; see chapter 4 in Kuznetsov et al., 2002. For a two-layer fluid, dipoles have already been obtained by Linton \& McIver (1995). For simplicity, attention is again restricted here to the limits in which the singular point approaches the interface between the two fluids. For a horizontal dipole on the upper side of the interface at $(x, y)=\left(\xi, 0_{+}\right)$, the potentials are

$$
G_{1}^{(1, j)}(x, y ; \xi)= \begin{cases}\frac{1}{\nu} f_{0}^{\infty}\left(A^{(1)}(k) \mathrm{e}^{k y}+\left[1+B^{(1)}(k)\right] \mathrm{e}^{-k y}\right) \sin k(x-\xi) \mathrm{d} k, & j=1 \\ \frac{1}{\nu} f_{0}^{\infty} C^{(1)}(k) \mathrm{e}^{k y} \sin k(x-\xi) \mathrm{d} k, & j=2 .\end{cases}
$$

The first index in the $G$ superscript refers to the layer in which the singularity lies, and the second index to the layer in which the particular expression is valid. Here

$$
\begin{gathered}
A^{(1)}(k)=\frac{[(\sigma+1) \nu-2 k](k+\nu) \mathrm{e}^{-2 k d}}{(k-\nu) h(k)}, \quad B^{(1)}(k)=-\frac{(k+\nu) \mathrm{e}^{-2 k d}+k-\nu}{h(k)}, \\
C^{(1)}(k)=-\frac{(\sigma-1) \nu B^{(1)}(k)}{k-\nu} .
\end{gathered}
$$

The corresponding stream functions are denoted by $H_{1}^{(1, j)}(x, y ; \xi), j=1,2$, and as $r \rightarrow 0$,

$$
H_{1}^{(1, j)}(x, y ; \xi) \sim \begin{cases}-2 \cos \theta /(\nu r)+(\sigma-1) \log r+O(1), & j=1, \\ \sim(\sigma-1) \log r+O(1), & j=2 .\end{cases}
$$

Thus, the solution is dipole-like on only the upper side of the interface, although it is still singular on the lower side of the interface with a vortex singularity.

For a horizontal dipole on the lower side of the interface at $(x, y)=\left(\xi, 0_{-}\right)$, the potentials in the upper and lower fluid layers are respectively

$$
G_{1}^{(2,1)}(x, y ; \xi)= \begin{cases}\frac{1}{\nu} f_{0}^{\infty}\left(A^{(2)}(k) \mathrm{e}^{k y}+B^{(2)}(k) \mathrm{e}^{-k y}\right) \sin k(x-\xi) \mathrm{d} k, & j=1 \\ \frac{1}{\nu} f_{0}^{\infty}\left[1+C^{(2)}(k)\right] \mathrm{e}^{k y} \sin k(x-\xi) \mathrm{d} k, & j=2,\end{cases}
$$

where

and

$$
A^{(2)}(k)=\frac{\nu(\sigma+1)(k+\nu) \mathrm{e}^{-2 k d}}{(k-\nu) h(k)}, \quad B^{(2)}(k)=\frac{\nu(\sigma+1)}{h(k)}
$$

$$
C^{(2)}(k)=-\frac{\left[(k+\sigma \nu) \mathrm{e}^{-2 k d}-k+\nu\right](k+\nu)}{(k-\nu) h(k)} .
$$

The corresponding stream functions are denoted by $H_{1}^{(2, j)}(x, y ; \xi), j=1,2$, and as $r \rightarrow 0$,

$$
H_{1}^{(2,1)}(x, y ; \xi) \sim \begin{cases}-(\sigma+1) \log r+O(1), & j=1, \\ -2 \cos \theta /(\nu r)-(\sigma+1) \log r+O(1), & j=2\end{cases}
$$

Now, the solution is dipole-like on only the lower side of the interface while, to leading order, there is a vortex singularity on the upper side of the interface. 


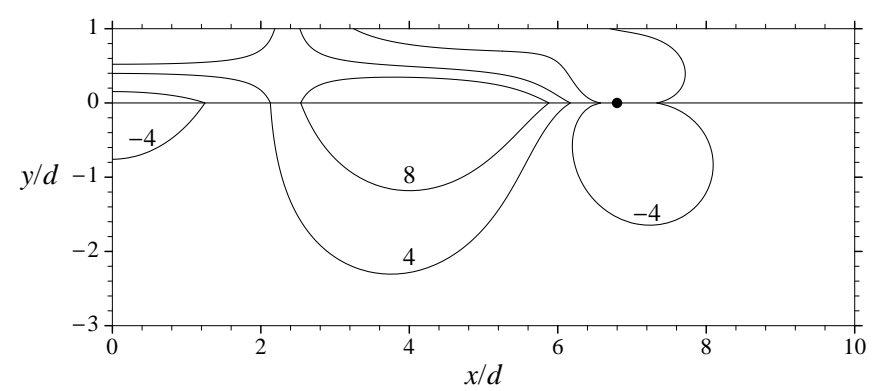

FiguRE 4. Streamline pattern for two dipoles on either side of interface; $\alpha=1, \beta=-1$, $m=1, n=0, \sigma=2$.

$$
\begin{aligned}
& \text { As } \nu|x| \rightarrow \infty \\
& \begin{aligned}
G_{1}^{(l, j)}(x, y ; \xi) \sim & \pi \operatorname{sgn} x\left[\mathrm{R}\left(C^{(l)}: \nu\right) \mathrm{e}^{\nu y} \cos \nu(x-\xi)\right. \\
& \left.+\mathrm{R}\left(C^{(l)}: \nu_{0}\right) \mathrm{e}^{\nu_{0} y}\left[\frac{\nu \sigma-\nu_{0}}{\nu(\sigma-1)}+\frac{\nu-\nu_{0}}{\nu(\sigma-1)} \mathrm{e}^{-2 \nu_{0} y}\right]^{2-j} \cos \nu_{0}(x-\xi)\right] .
\end{aligned}
\end{aligned}
$$

Trapped mode solutions can be found from potentials

$$
\begin{aligned}
\mathcal{U}_{ \pm}^{(j)}(x, y ; \xi)=\alpha\left[G_{1}^{(1, j)}(x, y ;-\xi)\right. & \left. \pm G_{1}^{(1, j)}(x, y ; \xi)\right] \\
& +\beta\left[G_{1}^{(2, j)}(x, y ;-\xi) \pm G_{1}^{(2, j)}(x, y ; \xi)\right], \quad j=1,2 .
\end{aligned}
$$

where $\alpha$ and $\beta$ are constants and, to annul the waves at infinity, $\xi$ is chosen in exactly the same way as described after equation (5.3). The corresponding stream functions $\mathcal{V}_{ \pm}^{(j)}$, $j=1,2$, are easily found. A local analysis based on the asymptotic forms in (5.6) and (5.8) indicates that streamlines enclosing the singularity are possible only if $\alpha$ and $\beta$ do not have the same sign and this has been confirmed in numerical calculations.

An example of a streamline pattern that may be obtained by the above dipole construction (with the dipoles added in each pair) is shown in Fig 4. The general comments about the source construction made above apply here also. Trapping structures constructed from dipole potentials in the lower fluid have a characteristic almost circular shape as typified by the streamline $\mathcal{V}_{+}=-4$.

\section{Conclusion}

In this paper the question of uniqueness of solution of the radiation and scattering problems in two-layer fluids has been studied and examples of bodies which support trapped modes have been constructed. Uniqueness of solution for a wide class of bodies was established with the use of a new form of Maz'ja's identity for a two-layer fluid. In particular, the classical result of John (1950), which states that no trapped modes can be supported by a single body which has the property that vertical lines drawn from every point on the free surface must not intersect the body, was extended to the configurations of bodies illustrated in example 2 .

However a general proof of uniqueness is not possible, and this was demonstrated by the 
direct construction of bodies which support trapped modes. An extension of the inverse procedure of McIver (1996) was used and bodies were formed from the streamlines of flow fields associated with singular solutions of the boundary value problem. In contrast to the situation for a single layer of fluid, none of the bodies which were constructed had a smooth boundary, but they all had discontinuous gradients at their intersection with the interface between the fluids.

This work was carried out during visits to Loughborough University by Dr. Kuznetsov. The financial support of NATO, the London Mathematical Society, and Loughborough University is gratefully acknowledged.

\section{REFERENCES}

Gorgui, M. A. \& Kassem, S. E. 1978 Basic singularities in the theory of internal waves. $Q . J l$ Mech. appl. Math. 46, 89-116.

John, F. 1950 On the motion of floating bodies, II. Comm. Pure Appl. Math. 3, 45-101.

Kuznetsov, N., Maz'Ya, V. \& Vainberg, B. 2002 Linear Water Waves: A Mathematical Approach. Cambridge University Press.

Linton, C. M. \& McIver, M. 1995 The interaction of waves with horizontal cylinders in two-layer fluids. J. Fluid Mech. 304, 213-219.

MCIVER, M. 1996 An example of non-uniqueness in the two-dimensional linear water wave problem. J. Fluid Mech. 315, 257-266.

WECK, N. 1990 On a boundary value problem in the theory of linear water waves Math. Meth. Appl. Sci. 12, 393-404. 